\title{
Didáctica de la Matemática y su importancia en los profesores en formación
}

\section{Didactic of Mathematics and its importance}

\section{in training teachers}

\section{Referencia del artículo}

\author{
Denisse Lorelei Monroy y Brenda Marroquín \\ - Facultad de Humanidades \\ Universidad de San Carlos de Guatemala \\ loreleimate@gmail.com
}

Monroy, D. y Marroquín, B. (2020). Didáctica de la Matemática y su importancia en los profesores en formación. Revista Guatemalteca de Educación Superior, 3(1), 47-59.

DOI: https://doi.org/10.46954/revistages.v1i1.4

Recibido 04/05/2020.

Revisado 05/05/2020.

Aceptado 25/06/2020.

\section{Resumen}

El artículo tiene como objetivo principal dar a conocer la importancia de la Didáctica de la Matemática en la formación del profesor que se especializa en la enseñanza de esta disciplina a nivel superior. El estudio tuvo un alcance descriptivo, con un diseño no experimental transversal; a través de un enfoque mixto con diseño concurrente. La fase cuantitativa fue no experimental, en la que se aplicaron cuestionarios tipo encuesta. La fase cualitativa fue fenomenológica, en la que se hizo una entrevista estructurada para recopilar información sobre el estudio. En la investigación participaron estudiantes, docentes y el coordinador de la cátedra de Matemática de la Escuela de Formación de Profesores de Enseñanza Media -EFPEM- de la Universidad de San Carlos de Guatemala -USAC-. Se utilizó un muestreo no probabilístico por conveniencia, buscando muestras homogéneas, sumando un total de setenta y cinco estudiantes, cinco docentes y el coordinador. 
Facultad de Humanidades

Escuela de Estudios de Postgrado

Palabras clave

Didáctica de la Matemática, formación, profesores, enseñanza, aprendizaje.
Keywords

mathematics teaching, training, teachers, didactics, learning.
Los resultados revelaron de forma general que la institución universitaria sí ha brindado una buena formación en didáctica a los estudiantes durante su carrera profesional; a pesar de que dentro del pensum de estudios solo reciben un curso de Didáctica especial de la Matemática. Se concluye con que la importancia que le brinda la institución descansa en el hecho de que no basta el conocimiento de una ciencia, sino que también es necesario el conocimiento de la Didáctica y otros conocimientos para facilitar el aprendizaje de la Matemática.

\section{Abstract}

The main objective of the article is to raise awareness of the importance of Mathematics Didactics in the training of teachers who specialize in teaching this discipline at a higher level. The study had a descriptive scope, with a non-experimental transversal design, through a mixed approach with concurrent design. The quantitative phase was non-experimental, in which survey questionnaires were applied. The qualitative phase was phenomenological, in which a structured interview was conducted to collect information about the study. Students, teachers and the coordinator of the Mathematics area of School of Training of Middle School Teachers -EFPEM- of the University of San Carlos de Guatemala -USAC- participated in the research. A non-probability sampling was used for convenience, looking for samples homogeneous, adding a total of 75 students, five teachers and the coordinator. The results revealed in a general way that the university institution has provided a good training in didactics to the students during their professional career. Even though within the Pensum of studies they only receive a special course in Mathematics Teaching. The conclusion is that the importance given to the institution lies in the fact that knowledge of a science is not enough, but that knowledge of Didactics and other knowledge is also necessary to facilitate the learning of Mathematics.

\section{Introducción}

La Matemática es considerada una ciencia exacta, por lo cual su enseñanza y aprendizaje en el ámbito educativo es un gran desafío por parte del profesor que la enseña y un gran reto para los estudiantes que la aprenden. 
Por otra parte, resulta muy difícil negar la importancia del aprendizaje de las matemáticas dentro de la formación escolar. Académicos de diversas disciplinas, así como personas vinculadas directamente a la educación y a las matemáticas insisten en que el aprendizaje y comprensión de la misma es un factor clave para el éxito tanto escolar como personal (Castillo, 2016).

Hay que resaltar que a pesar de la importancia del aprendizaje de la Matemática, muchos estudiantes la consideran difícil de aprender. Además, las evaluaciones que se aplican y se han aplicado en Guatemala, tanto en el marco de estudios internacionales como evaluaciones nacionales, muestran sistemáticamente resultados insatisfactorios en el ár matemática, lo que parece indicar que el sistema educativo enfrenta serias limitaciones para formar las competencias que los niños y jóvenes requieren para ella. A nivel universitario, el área de matemáticas es la que reporta menores índices de aprobación en pruebas de admisión, por lo que se puede asumir que es el área menos desarrollada en los jóvenes aspirantes a la universidad (Castillo, 2016).

Un elemento central ante esta problemática, es la formación del profesor que se especializa en la enseñanza de la Matemática; ya que la calidad de la educación depende, en gran medida, de la formación docente y de cómo dirige y orienta el proceso de enseñanza-aprendizaje" (Torres, 2009).

Ante esto, es fundamental que los futuros profesores que se especializan en la enseñanza de la Matemática a nivel superior adquieran en su proceso de formación no solo los conocimientos propios de la ciencia; sino que también adquieran conocimientos didácticos, pedagógicos y conocimientos de cómo aprende el estudiante, según las características particulares de la persona y del nivel educativo; los cuales deben estar acordes con la naturaleza de la especialidad que se va a enseñar, en este caso de la Matemática.

Lo cual significa que un aspecto clave en la formación del profesor es la Didáctica en este caso enfocada en el área de la Matemática, es decir, la Didáctica de la Matemática. Ante esto, hay que resaltar que la Didáctica por sí sola tiene como campo de estudio "la enseñanza", además de otros elementos que la integran como 
Facultad de Humanidades

Escuela de Estudios de Postgrado los métodos, técnicas, procedimientos, estrategias y recursos didácticos acordes con la naturaleza del conocimiento que se ha de enseñar, en este caso con la Matemática y las particularidades socio-cognitivas del estudiante al que le enseña (Herrera, 1999).

En otro sentido, la Didáctica de la Matemática "se ocupa de indagar metódica y sistemáticamente sobre los procesos de enseñanza y aprendizaje de las matemáticas así como de los planes para la preparación profesional de los educadores matemáticos" (Rico, Sierra, \& Castro, 2002).

Lo cual resalta la importancia que "la formación de profesores de matemáticas como contexto práctico tiene de referente teórico a la Didáctica de la Matemática (DM) como dominio científico" (Llinares, Sánchez, García, \& Escudero, s/f: 211). Es importante resaltar el compromiso que tienen las instituciones universitarias que forman a profesores especializados en la Matemática de velar por la calidad profesional de sus egresados. Las competencias básicas para la formación del profesor que se especializa en la enseñanza de la Matemática se deben adquirir en la Universidad ya que, como campo profesional propio de los grados universitarios, estas competencias básicas deben estar vinculadas a la formación de grado o de postgrado (Rico, 2004).

Dado que en Guatemala la Escuela de Formación de Profesores de Enseñanza Media -EFPEM- de la Universidad de San Carlos de Guatemala -USAC- es una institución educativa pública a nivel Superior que forma a profesores especializados en la Matemática se realizó una investigación con diseño descriptivo que plantea como pregunta principal:

¿Cuál es la importancia que se le brinda a la Didáctica de la Matemática en la formación del profesor especializado en la enseñanza de la Matemática a Nivel Superior en la EFPEM?

Dicha investigación tuvo como objetivo general: determinar la importancia que se le brinda a la Didáctica de la Matemática en la formación del profesor especializado en la enseñanza de la Matemática a Nivel Superior en la EFPEM, y para esto se plantearon como objetivos específicos: analizar la percepción de estudiantes y 
docentes respecto a la importancia de la didáctica en su formación profesional; establecer qué tipo de formación didáctica tiene el profesor que se especializa en la enseñanza de la Matemática a Nivel Superior e identificar qué elementos de la didáctica aplican los docentes que imparten Matemática en la EFPEM.

\section{Materiales y métodos}

En la investigación la población estuvo constituida por estudiantes del primer semestre de la Licenciatura en la Enseñanza de la Matemática y Física del Plan Diario y del Plan Sabatino de la Escuela de Formación de Profesores de Enseñanza Media EFPEM- de la Universidad de San Carlos de Guatemala, con una población aproximada de ciento cinco estudiantes, cinco docentes que imparten cursos de Matemática y Didáctica de la Matemática en ambos planes de estudio y el coordinador de la cátedra de Matemática.

Se utilizó un muestreo no probabilístico por conveniencia, buscando muestras homogéneas. Lo cual significa que se tomaron solo los casos disponibles a los cuales se tenía acceso, sin embargo, dichas unidades poseían un mismo perfil o características similares. Estas características fueron: ser estudiante inscrito en el ciclo 2020 de la Universidad de San Carlos de Guatemala, cursar el primer semestre de la Licenciatura en Matemática y Física, ser graduados o con cierre de pensum de la carrera de Profesorado de Enseñanza Media en Matemática y Física de la institución; considerando que dichos estudiantes ya tienen una perspectiva clara de cómo fue su formación profesional en el profesorado; sumando un total de setenta y cinco estudiantes.

Así mismo, la investigación tuvo un alcance descriptivo, con un diseño no experimental transversal. El enfoque fue mixto, a través de un diseño concurrente. La fase cuantitativa fue no experimental en la que aplicaron cuestionarios tipo encuesta. La fase cualitativa fue fenomenológica en la que se hizo una entrevista estructurada para recopilar información sobre el fenómeno estudiado.

Los instrumentos para los estudiantes se aplicaron durante el horario de clases con previa autorización. Los instrumentos para 
los docentes y para el coordinador de la cátedra de Matemática fueron aplicados de forma individual en el salón de profesores del área de Matemática de la institución. Además, como parte de las consideraciones éticas de la investigación, se solicitó el consentimiento informado de los participantes antes de la aplicación de los instrumentos.

Los datos cuantitativos que se obtuvieron de la encuesta sociodemográfica a docentes y estudiantes y de la encuesta sobre el tema de estudio a docentes y estudiantes, se tabularon en una base de datos en el programa Excel. Se utilizó la estadística descriptiva para reportar los datos en términos de frecuencias (porcentajes) en tablas y gráficas. Luego se realizó la interpretación de resultados de cada una.

Para los datos cualitativos obtenidos de la entrevista se realizó un análisis de contenido a través de palabras clave o categorías para la interpretación y el análisis previo. Además, se realizó una triangulación y un contraste de los resultados obtenidos de las encuestas sobre el tema de estudio de los docentes, con los resultados de la de los estudiantes y de los resultados obtenidos en la entrevista.

\section{Resultados}

Para caracterizar la muestra de estudio se aplicó una encuesta sociodemográfica tanto a estudiantes como a docentes, los principales datos que se obtuvieron se presentan a continuación.

Respecto a los estudiantes es importante resaltar que el $77 \%$ trabajan como profesores, el 68\% tiene cierre de pensum de Profesor de Enseñanza Media en Matemática y Física y un 32\% están graduados. Otro dato muy interesante es que la formación de la población a Nivel Medio refleja que el $45 \%$ de la población son bachilleres y solo el $40 \%$ son maestros, lo cual evidencia la población que tiene conocimientos previos en Didáctica y Pedagogía antes de iniciar con su formación como Profesor de Enseñanza Media. 
Respecto a la información obtenida de la encuesta sociodemográfica a los docentes, se resalta que el $100 \%$ de los docentes son hombres, de lo cual se deduce la falta de participación de mujeres docentes en el área de Matemática. De los docentes, el $80 \%$ tiene doble jornada de trabajo tanto en plan Diario como en plan Sabatino, impartiendo un promedio de dos a tres cursos. Con un rango de experiencia docente de dos a treinta años. Con reslpecto al nivel académico de los docentes encuestados, el $60 \%$ tiene una maestría y también solo el $60 \%$ posee la Licenciatura en la Enseñanza de la Matemática. Estos datos fueron importantes para la investigación porque reflejan el perfil del docente.

En cuanto a los resultados de la encuesta sobre el tema aplicada, tanto a estudiantes como a docentes, y de la entrevista realizada al coordinador de la cátedra de Matemática, se presentan los siguientes resultados de acuerdo con cada uno de los objetivos específicos planteados.

Tabla 1. Percepción respecto a la importancia que se le brinda a la didáctica de la Matemática en la formación profesional

\begin{tabular}{|l|c|c|}
\hline \multicolumn{1}{|c|}{ Indicadores } & $\begin{array}{c}\text { Promedio (f\%) según los } \\
\text { estudiantes }\end{array}$ & $\begin{array}{c}\text { Promedio (f\%) según los } \\
\text { docentes }\end{array}$ \\
\hline Siempre & $53 \%$ & $63 \%$ \\
\hline Casi siempre & $32 \%$ & $17 \%$ \\
\hline A veces & $12 \%$ & $17 \%$ \\
\hline Nunca & $3 \%$ & $3 \%$ \\
\hline Totales & $100 \%$ & $100 \%$ \\
\hline
\end{tabular}

Fuente: Monroy (2020).

Según los resultados obtenidos de la tabla uno sobre la percepción, tanto de los estudiantes como de los docentes, se deduce que en la formación profesional del estudiante sí se le da importancia a la Didáctica enfocada en la enseñanza de la Matemática. Según la información obtenida en la entrevista, esto se debe a la naturaleza de la carrera que es en enseñanza, por lo cual la institución sí le brinda la debida importancia a la Didáctica de la Matemática en la formación del profesor que se especializa en esta área de enseñanza. 
Tabla 2. Tipo de formación didáctica del profesor que se especializa en la enseñanza de la Matemática a Nivel Superior en la EFPEM

\begin{tabular}{|l|c|c|}
\hline Indicadores & $\begin{array}{l}\text { Promedio (f\%) según los } \\
\text { estudiantes }\end{array}$ & $\begin{array}{c}\text { Promedio (f\%) según los } \\
\text { docentes }\end{array}$ \\
\hline Siempre & $40 \%$ & $68 \%$ \\
\hline Casi siempre & $36 \%$ & $30 \%$ \\
\hline A veces & $20 \%$ & $2 \%$ \\
\hline Nunca & $4 \%$ & $0 \%$ \\
\hline Totales & $100 \%$ & $100 \%$ \\
\hline
\end{tabular}

Fuente: Monroy (2020).

Según la tabla dos, el $40 \%$ de los estudiantes considera que siempre tuvieron una buena formación en didáctica durante su carrera profesional y el $36 \%$ indican que casi siempre; a pesar que el $68 \%$ de los docentes que imparten los cursos consideran que siempre brindaron una buena formación en Didáctica. Por otra parte, en la entrevista realizadase afirma que la institución sí le brinda importancia tanto a la formación del conocimiento didáctico como al conocimiento matemático, a pesar de que solo cuentan con un curso de Didáctica especial de la Matemática; se evidenció que reciben otros cursos que complementan dicha formación en Didáctica, lo cual fue comprobado al realizar una revisión al pensum de estudios de la carrera.

Tabla 3. Elementos de la didáctica aplicados por los docentes que imparten Matemática en la EFPEM

\begin{tabular}{|l|c|c|}
\hline \multicolumn{1}{|c|}{ Indicadores } & $\begin{array}{c}\text { Promedio }(\mathbf{f} \%) \text { según los } \\
\text { estudiantes }\end{array}$ & $\begin{array}{c}\text { Promedio (f } \%) \text { según } \\
\text { los docentes }\end{array}$ \\
\hline Siempre & $37 \%$ & $80 \%$ \\
\hline Casi siempre & $42 \%$ & $20 \%$ \\
\hline A veces & $18 \%$ & $0 \%$ \\
\hline Nunca & $3 \%$ & $0 \%$ \\
\hline Totales & $100 \%$ & $100 \%$ \\
\hline
\end{tabular}

Fuente: Monroy (2020). 
Según la tabla tres, los estudiantes indican que el $37 \%$ de sus docentes que les imparten cursos siempre hacen uso adecuado de los elementos didácticos; sin embargo, un $42 \%$ indicaron que casi siempre. Entre los elementos que aplican están: el uso de recursos didácticos, la aplicación de diversas actividades de aprendizaje, utilización de diferentes herramientas de evaluación, entre otros. Esto contradice los resultados según los docentes, ya que el $80 \%$ indicaron que siempre hacen uso adecuado de los elementos didácticos como los mencionados anteriormente.

\section{Discusión}

En respuesta a la pregunta planteada al inicio del estudio ¿Cuál es la importancia que se le brinda a la Didáctica de la Matemática en la formación del profesor especializado en la enseñanza de la Matemática a Nivel Superior en la EFPEM? Se determinó a partir de la información obtenida que en la formación profesional de los profesores de enseñanza Media en Matemática y Física de la EFPEM; sí se le brinda importancia a la Didáctica de la Matemática.

Así mismo, se determinó que este resultado se debe a la misma naturaleza de la carrera, porque es en enseñanza y por consiguiente si la institución solo les brindara los conocimientos matemáticos y no las herramientas didácticas y pedagógicas cometería un gran error y no se estaría cumpliendo con la misión de la institución que dice literalmente que es “...la responsable de la formación de profesionales de alta calidad, en el campo de la docencia, en los distintos niveles del sistema educativo nacional, y que, basados en la investigación, la docencia y la extensión, contribuimos al fortalecimiento del proceso educativo en el ámbito nacional..." (Escuela de Formación de Profesores de Enseñanza Media - EFPEM ,- 2019).

Además, se concluye que la diferencia de un profesor que se especializa en la enseñanza de la Matemáticas y del profesional que estudia la ciencia pura de la matemática radica en la importancia que se le da a la Didáctica de la Matemática en la formación profesional de dicha carrera. Por lo tanto, la importancia que le brinda la institución descansa en el hecho de que no basta el conocimiento de una ciencia, en este caso de la Matemática, 
sino que también es necesario el conocimiento didáctico; porque esta le proporciona las herramientas necesarias para facilitar el aprendizaje de la Matemática.

Esto se debe a que existen profesionales que tienen profundos conocimientos matemáticos, pero no tienen la didáctica para explicar los temas y según información obtenida, al carecer el profesor de conocimiento didácticos y pedagógicos son los estudiantes los que sufren, porque no comprenden las matemáticas; generando tensión, estrés, rechazo a las matemáticas a lo largo de la vida de la persona.

Al establecer qué tipo de formación didáctica tiene el profesor que se especializa en la enseñanza de la Matemática a nivel superior, se determinó de forma general que la institución universitaria sí ha brindado una buena formación en didáctica a los estudiantes durante su carrera profesional. A pesar de que dentro del pensum de estudios solo reciben un curso de Didáctica especial de la Matemática.

Sin embargo, se evidenció que llevan otros cursos que complementan esa formación del conocimiento didáctico de los estudiantes de dicha carrera, entre estos cursos están: Pedagogía I, II, principios de la Psicología del aprendizaje, Evaluación escolar I, II, Didáctica general, Inducción a la docencia I, II, Practica docente, la Educación Media y la formación integral del adolescente, que son cursos importantes en el ejercicio de la docencia.

Por otra parte, se evidenció en el pensum de estudios la cantidad de créditos de la carrera; en la parte de la formación enfocada en la cultura psicopedagógica, el estudiante adquiere 24 créditos, en la cultura profesional o específica de la carrera adquiere 72 créditos, en el área de formación de práctica docente adquiere 16 créditos y en el área de seminario que sería la parte investigativa de la carrera adquiere cinco créditos.

Por lo tanto, se concluye que según el análisis realizado al ensum de estudios y según los resultados de la entrevista realizada, el tipo de formación didáctica que adquieren los estudiantes de la carrera de Profesores de Enseñanza Media de la EFPEM, incluye los 
conocimientos propios de la ciencia, los conocimientos didácticos, conocimientos pedagógicos, conocimientos de los procesos de aprendizaje del estudiante y conocimientos en investigación.

Además, al identificar qué elementos de la didáctica aplican los docentes que imparten Matemática en la EFPEM, primero se determinó de forma general que los docentes si aplican los elementos didácticos en el ejercicio de su profesión como formador de formadores, sin embargo, algunos no los aplican de forma adecuada generando algún tipo de dificultad en el proceso de formación de los estudiantes del Profesorado de Enseñanza Media en Matemática.

Respecto a los elementos didácticos que aplican los docentes se identificaron los siguientes: la planificación de las sesiones de aprendizaje del curso que imparten, el desarrollo de varias actividades de aprendizaje, el uso de diversos tipos de recursos didácticos en el proceso de enseñanza de la Matemática y el uso de distintas técnicas para evaluar el aprendizaje de la Matemática. Esto según la información obtenida en la encuesta realizada tanto a docentes como a estudiantes y en la entrevista realizada.

Por último, es necesario resaltar que los docentes que son formadores de formadores tienen una doble responsabilidad, porque deben proporcionarles a los estudiantes las herramientas necesarias para que hagan efectivo el proceso de enseñanza y aprendizaje de la Matemática al ejercer la docencia. Lo anterior toma importancia porque muchas veces la forma en que les enseñan sus docentes durante su formación influye en la forma en que enseñarán los estudiantes que se están formando como profesores, ya que de alguna manera el estudiante no solo aprende con el docente los conocimientos que le imparte; sino también aprende de él algunos elementos de la metodología, estrategias, técnicas, actividades, procesos de evaluación, entre otros.

Ante esto, se recomienda de manera general a las instituciones universitarias que forman a profesores especializados en Matemática ofrecer calidad educativa en sus programas académicos y a velar porque estén actualizados de acuerdo a las demandas educativas actuales. 


\section{Agradecimientos}

Se agradece a las autoridades y docentes de la Escuela de Formación de Profesores de Enseñanza Media -EFPEM- de la Universidad de San Carlos de Guatemala por su apoyo y apertura para la realización de la investigación. Así mismo, se extiende un especial agradecimiento a los y las estudiantes y docentes que participaron en la muestra, por su disposición y confianza; especialmente al coordinador de la cátedra de Matemática por su valiosa colaboración y aporte a la investigación.

\section{Referencias}

Castillo, W. (2016). Así estamos enseñando matemáticas.

Ministerio de Educación de Guatemala, Dirección General de Evaluación e Investigación Educativa. Guatemala: divulgacion_digeduca@mineduc.gob.gt.

Escuela de Formación de Profesores de Enseñanza Media EFPEM -. (2019). Sitio Web https://www.efpemusac.org/ quienes-somos

Herrera, D. (1999). La didáctica universitaria: Referencia imprescindible para una enseñanza de calidad. Revista electrónica interuniversitaria de formación del profesorado, 2 (1).

Llinares, S., Sánchez, V., García, M., \& Escudero, I. (s/f). Didáctica de la Matemática y la formación de profesores de matemáticas de Enseñanza Secundaria. Las matemáticas del siglo XX: una mirada en, 101 (42), 211-214.

Navarra, J. (2001). Didáctica: concepto, objeto y finalidades. Universidad Nacional de Educación a Distancia, UNED. Didáctica general para psicopedagogos. 
Rico, L. (2004). Reflexiones sobre la formación inicial del profesor de matemáticas de secundaria. Profesorado. Revista de currículum y formación del profesorado, 8 (1), 1-15.

Rico, L., Sierra, M., \& Castro, E. (2002). El área de conocimiento de "Didáctica de la Matemática". Revista de Educación, 35-58.

Torres, H. (2009). Didáctica General. San José, Costa Rica: Coordinación Educativa y Cultural Centroamericana, CECC/SICA.

\section{Sobre la autora}

Denisse Lorelei Monroy Salguero es licenciada en la enseñanza de la Matemática y Física. Estudios de maestría en docencia universitaria. Actualmente trabaja en docencia a nivel primario, medio y universitario en Guatemala.

\section{Copyright (c) Denisse Lorelei Monroy y Brenda Marroquín}

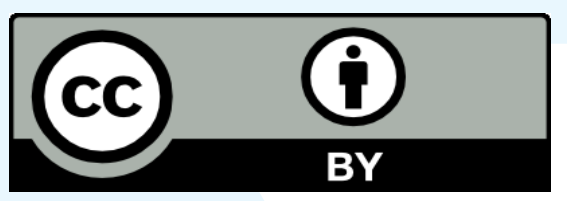

Este texto está protegido por una licencia CreativeCommons 4.0.

Usted es libre para compartir, copiar y redistribuir el material en cualquier medio o formato y adaptar el documento, remezclar, transformar y crear a partir del material para cualquier propósito, incluso comercialmente, siempre que cumpla la condición de atribución: usted debe reconocer el crédito de una obra de manera adecuada, proporcionar un enlace a la licencia, e indicar si se han realizado cambios. Puede hacerlo en cualquier forma razonable, pero no de forma tal que sugiera que tiene el apoyo del licenciante o lo recibe por el uso que hace. 